\title{
ANTIFUNGAL ACTIVITY OF KOMBUCHA TEA AGAINST HUMAN PATHOGENIC FUNGI
}

\author{
ARI YUNIART0 ${ }^{1 *}$, KUSNANDAR ANGGADIREDJA ${ }^{2}$, RIANA ANNISA NUR AQIDAH ${ }^{1}$
}

${ }^{1}$ Department of Pharmacology Research Group, Pharmacology Research Group, Bandung School of Pharmacy, Jl. Soekarno - Hatta No. 754, Bandung, West Java, Indonesia. ${ }^{2}$ Department of Pharmacology Research Group, Pharmacology and Clinical Pharmacy Research Group, School of Pharmacy, Bandung Institute of Technology (ITB), Jl. Ganeca 10, Bandung, West Java, Indonesia. Email: ariyuniarto@yahoo.co.id

Received: 11 June 2016, Revised and Accepted: 16 June 2016

\section{ABSTRACT}

Objective: In general, infectious diseases still known as a major contributor to the number of morbidity and mortality in the worldwide beside other diseases. Infections are ordinarily caused by viruses, bacteria, and fungi. Several human pathogenic fungi involving Aspergillus flavus, Candida albicans, and Microsporum gypseum have responsible against infectious diseases. The objective of the research was to evaluate the antifungal activity of kombucha tea against human pathogenic fungi such as A. flavus, C. albicans, and M. gypseum.

Methods: The antifungal activity of the kombucha tea against A. flavus, C. albicans, and M. gypseum were tested by disc diffusion method with duration of fermentation in 6,12 , and 18 days.

Results: Based on the present study, inhibitory diameter of A. flavus, C. albicans, and M. gypseum are 16.83, 15.36, and 25.06 mm, respectively. The inhibitory diameter was obtained from kombucha tea activity with duration of fermentation in 6 days.

Conclusion: The results provide support for the use of kombucha tea as a potential antifungal source against human pathogenic fungi involving A. flavus, C. albicans, and M. gypseum.

Keywords: Antifungal, Kombucha tea, Pathogenic, Fungi.

(C) 2016 The Authors. Published by Innovare Academic Sciences Pvt Ltd. This is an open access article under the CC BY license (http://creativecommons. org/licenses/by/4. 0/) DOI: http://dx.doi.org/10.22159/ajpcr.2016.v9i5.13432

\section{INTRODUCTION}

Infectious diseases still known as a leading problem occurred in several countries. Infections are the invasion mechanism of the host by the microorganism. Infections are ordinarily caused by fungi, bacteria, and viruses [1]. In fact, infectious diseases still ranked as the major contributor to the number of morbidity and mortality in the worldwide including in Indonesia. Typical of fungal infections which take place in Indonesia are triggered by various factors. They are tropical climate conditions, which maintain the fungal growth, poor personal sanitation, and hygiene, and inadequate immunity (immunocompromised patients such as AIDS, cancer diseases, or organ transplantation) [2-4].

Pathogenic fungi, which predominantly attack humans, are Microsporum gypseum that provokes skin disease; Candida albicans that induce candidiasis, and Aspergillus flavus that leads to Aspergillosis. The value of incident of fungal infections triggered by Microsporum has reached 1.5 billion cases per year as well as the sickness which caused by $C$. albicans and $A$. flavus that count 9.5 million and 1 million cases per year, respectively [5].

Meanwhile, fungal infections management can be executed in both nonpharmacological and pharmacological treatment. Non-pharmacological treatment can be completed by maintaining personal hygiene and protect the immune system. At the same time, pharmacological treatment can be executed by administering proper antifungal drugs based on the cause of the infecting fungi. The use of antifungal drugs which incorrectly give contributions for the resistance of the fungi. Therefore, it is required to develop potential antifungal that effectively eradicate the fungi such as kombucha tea.

Kombucha is a beverage of a fermented tea consumed traditionally in many countries and have several benefits. Kombucha obtained by fermenting sweetened tea and tea fungus. It is recognized as symbiotic culture of bacteria and yeast which forms as gelatin [6-8]. Tea fungus is a symbiotic culture of acetic acid bacteria (Acetobacter aceti, Acetobacter pasteurianus, and Gluconobacter oxydans) and yeasts (Saccharomyces sp., Zygosaccharomyces kombuchaensis, and Torulopsis sp.) [9]. This jelly form has brownish-white, springy texture, and immense water content. This symbiosis results acid and alcohol that interfere the advancement of other microorganisms that are inhibited from the kombucha tea. The objective of the present study was to evaluate the antifungal activity of kombucha tea against human pathogenic fungi such as A. flavus, C. albicans, and M. gypseum.

\section{METHODS}

Fungal specimens and preparation of inoculum

Fungal specimens that used in this study such as A. flavus, C. albicans, and $M$. gypseum. The fungal specimens were grown at $25^{\circ} \mathrm{C}$ on sabouraud dextrose agar (SDA) medium for 24-72 hrs.

\section{Preparation of fungal suspension}

Fungal specimens suspended in liquid media of $0.9 \%$ sodium chloride. It is incubated for $18-24 \mathrm{hrs}$ at $25^{\circ} \mathrm{C}$. Furthermore, shake the suspension and control the turbidity to get close to the standard of turbidity, 0.5 McFarland. The standard of turbidity, $0.5 \mathrm{McF}$ arland, is equal to the fungal content which counts $10^{8}$ colony forming unit $/ \mathrm{ml}$. It has absorbance in the value of $0.08-0.10$ in wavelength of $530 \mathrm{~nm}$.

\section{Kombucha culture rejuvenation}

The aim of this procedure is to establish kombucha culture which will be inoculated into fermentation media. Kombucha culture rejuvenation was started with preparing the fermentation media by heating of $1000 \mathrm{ml}$ aquadest in a sterilized beaker glass until temperature of $100^{\circ} \mathrm{C}$ for \pm 15 minutes, followed by the addition of $0.5 \%$ black tea. Then, the mixture was filtered. Results of black tea involving residue and filtrate which contributes as the growth media of kombucha. The filtrate was measured to $600 \mathrm{ml}$, added with $10 \%$ sucrose, 
and diluted until homogeneous. The solution is packed into a glass bottle. Furthermore, the media is cooled down to room temperature $\left(25^{\circ} \mathrm{C} \pm 2^{\circ} \mathrm{C}\right)$. Progressing into the next stage, inoculation stage, the prepared media is combined with kombucha culture $10 \%$. Hereafter the bottle is covered with porous cloth and sealed with rubber. Then, it is located in a stable area, which is protected from sunrays. The result of this rejuvenation of kombucha tea culture is ready for the final step which is the fermentation for 18 days

\section{Measurement of antifungal activity}

Antifungal activity of kombucha tea was tested by the paper disc method. First, the materials and tools are prepared into laminar air flow. Heat the SDA until it melts. Cool it down to $40-50^{\circ} \mathrm{C}$. Then, add $1 \mathrm{ml}$ of fungal suspension specimen. Add about $20 \mathrm{ml}$ of cooled SDA to the Petri plates. Let the mixture of mold suspension and the media is solidified. Prepare the paper disc and the liquid which is originated from the fermentation of kombucha tea. On the paper disc, using sterilized tweezers, place 3 drops of $20 \mu \mathrm{l}$ of the liquid aseptically $(1.5 \mathrm{~cm}$ from the side of Petri plates). This medium is then incubated at room temperature for $3 \times 24$ hrs. The antifungal activity is displayed by the inhibition zone of the specimen around the paper disc.

\section{Measurement of the equivalence of kombucha tea solution and} miconazole

The test of equivalence of kombucha tea and antifungal agent miconazole is executed by paper disc method. From the primary solution of $20,000 \mu \mathrm{g} / \mathrm{ml}$, they were created a series of solution with distinctive concentration from 18,000, 16,000, 14,000, 12,000, 10,000 8000,6000 , and $5000 \mu \mathrm{g} / \mathrm{ml} .1 \mathrm{ml}$ of fungal suspension is added into sterilized Petri plates. Add $\pm 20 \mathrm{ml}$ of liquid sterilized SDA. Furthermore, shake the Petri plates to homogenize the mixture of the media and the suspension. When it solidifies, aseptically add three paper disc by distance of $1.5 \mathrm{~cm}$ from the side of Petri plates. Ensure that the paper disc is added with $20 \mu \mathrm{l}$ of diluted miconazole in dimethyl-sulfoxide. Hereafter, it is incubated in $25^{\circ} \mathrm{C}$ for $24-72$ hrs. Subsequently, it measures the inhibitory diameter.

\section{Statistical analysis}

Statistical analysis of the result was performed using the one-way analysis of variance, followed with the post-hoc test Tukey honestly significant difference. A value of $\mathrm{p}<0.05$ was used to explain data significance.

\section{RESULTS AND DISCUSSION}

Kombucha tea is a traditional beverage originated from the fermentation of tea and sugar. It has unique aromatic, sweet, and sour taste and contains diversified type of vitamin, minerals, and organic acid of fermented tea leaves. Kombucha is the results of fermentation of yeast culture and acid bacteria. The fermentation process of kombucha is affected by several factors of surroundings such as amount of inoculum, incubation temperature, $\mathrm{pH}$, and initial sugar concentration $[10,11]$. In kombucha fermentation, tea is used as the fermentation media Tea has functioned as mineral supply for the reaction of yeast and bacteria. Besides, it takes a role in giving the flavor and specific aroma of the kombucha. In this research, it used black tea which commonly consumed because its polyphenol oxidation helps conveying the strong color and unique taste. Black tea produces more metabolic material compared to other media [12].

Antifungal activity test of kombucha tea was performed by diffusion method using paper disc method against human pathogenic fungi such as A. flavus, C. albicans, and M. gypseum. In this research, the fungi were performed as it represents the three mycosis infections consist of cutaneous mycosis (M. gypseum), mucosal mycosis (C. albicans), and systemic mycosis (A. flavus). In the test of the medium, SDA is applied due to its objective as a specific media for the fungal growth.

Test of inhibitory activity of the fungi aims to determine the ability of the product (the result of kombucha tea fermentation) in inhibiting the growth of pathogenic fungi such as A. flavus, C. albicans, and M. gypseum. The activity test is accomplished in 6,12 , and 18 days.

According to Table 1, test of inhibitory activity of kombucha tea against fungal specimen in the time of 6,12 , and 18 days exhibit different inhibitions regarding the obtained inhibition zone. This phenomenon indicates that, in those fermentation conditions, kombucha tea genuinely has antifungal activity. As the duration of fermentation is increased, the condition of the media is getting acid.

In the present study, it reveals that the increase of fermentation period will diminish the antifungal activity. It is supported with the data that there is a declining trend of inhibitory diameter during 6,12, and 18 days of fermentation. It is expected that in the 6 days of fermentation, the yield of metabolic compound is greater than the yield of 12 and 18 days fermentation, as well as having higher ability in impeding the growth of fungi. The probable compounds that perform as the antifungal agent are acetic acid, lactic acid, ethanol, glucuronic acid, which originated from the fermentation of kombucha.

Acetic acid or $\mathrm{CH}_{3} \mathrm{COOH}$ is a clear fluid that has sour taste and pungent odor. Acetic acid is weakly ionized in aqueous solution and historically used as preservative due to its antimicrobial effect [13]. Organic acid (acetic acid) can be applied as antimicrobial compound as it suppresses the growth of pathogenic microbes by deactivating the work system of the cell: Cell wall, cell membrane, metabolic enzymes, and protein synthesis system.

In positive control test, miconazole has a role as the antifungal agent. Miconazole is derived from synthetic imidazole which relatively stable and has broad antifungal spectrum against dermatophyte fungi. Miconazole inhibits the fungal activity of Trichophyton, Epidermophyton Microsporum, Candida, and Malassezia furfur [14]. Moreover, dimethylsulfoxide is used as the solvent of miconazole which acts as a negative control. As tea is known for its antimicrobial activity, however, the 5\% concentration of black tea which is used as the negative control results no inhibition of the fungal growth.

From the Fig. 1, it showed that inhibitory diameter of kombucha tea in 6 days of fermentation, gives the equivalence value of $20 \mu \mathrm{l}$ kombucha tea and miconazole: $5696 \mu \mathrm{g} / \mathrm{ml}$ for A. flavus, $5487.38 \mu \mathrm{g} / \mathrm{ml}$ for C. albicans, and $9174.44 \mu \mathrm{g} / \mathrm{ml}$ for M. gypseum, respectively.

Table 1: The average of inhibitory diameter ( $\mathrm{mm}$ ) of kombucha tea against $A$. flavus, $C$. albicans, and M. gypseum

\begin{tabular}{llll}
\hline Duration (days) & \multicolumn{4}{l}{ Average of inhibitory diameter (mm) } \\
\cline { 2 - 4 } & A. flavus & C. albicans & M. gypseum \\
\hline 6 & $16.83 \pm 0.05$ & $15.36 \pm 0.87$ & $25.06 \pm 0.32$ \\
12 & $15.43 \pm 0.25$ & $14.56 \pm 0.98$ & $22.50 \pm 0.75$ \\
18 & $11.06 \pm 0.35^{*}$ & $11.00 \pm 0.81^{*}$ & $21.16 \pm 0.90^{*}$ \\
\hline
\end{tabular}

*Data represent the mean \pm SD of observations, significant different during fermentation period $\mathrm{p}<0.05$. SD: Standard deviation, A. flavus: Aspergillus flavus, C. albicans: Candida albicans, M. gypseum: Microsporum gypseum

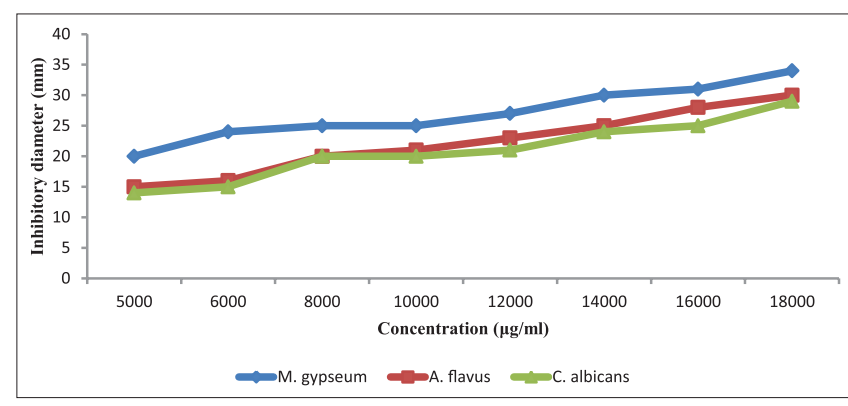

Fig. 1: Antifungal activity of miconazole against inhibitory diameter of fungi 


\section{CONCLUSION}

The results from the present study provide support for the use of kombucha tea as a potential antifungal source against human pathogenic fungi involving A. flavus, C. albicans, and M. gypseum.

\section{REFERENCES}

1. Crum-Cianflone NF. Bacterial, fungal, parasitic, and viral myositis. Clin Microbiol Rev 2008;21(3):473-94.

2. Blanco JL, Garcia ME. Immune response to fungal infections. Vet Immunol Immunopathol 2008;125(1-2):47-70.

3. Samonis G, Bafaloukos D. Fungal infections in cancer patients: An escalating problem. In Vivo 1992;6(2):183-93.

4. Silveira FP, Husain S. Fungal infections in solid organ transplantation. Med Mycol 2007;45(4):305-20.

5. Vandeputte P, Ferrari S, Coste AT. Antifungal resistance and new strategies to control fungal infections. Int J Microbiol 2012;2012:713687.

6. Jayabalan R, Malbasa RV, Loncar ES, Vitas JS, Sathiskumar M. A review on kombucha-microbiology, composition, fermentation, and beneficial effects, toxicity, and tea fungus. Compr Rev Food Sci Food Saf 2014;13:538-50.

7. Essawet NA, Cvetkovic D, Velicanski A, Canadanovic-Brunet J,
Vulic J, Maksimovic V, et al. Polyphenol and antioxidant activities of kombucha beverage enriched with coffeeberry ${ }^{\circledR}$ extract. Chem Chem Eng Q 2015;21(3):399-409.

8. Goh WN, Rosma A, Kaur B, Fazilah A, Karim AA, Rajeev B. Fermentation of black tea broth (Kombucha): I. Effects of sucrose concentration and fermentation time on the yield of microbial cellulose. Int Food Res J 2012;19(1):109-17.

9. Velicanski A, Cvetkovic D, Markov S. Characteristics of kombucha fermentation on medicinal herbs from lamiaceae family. Rom Biotechnol Lett 2013;18(1):8034-42.

10. Frank GW. Kombucha: Healthy Beverage and Natural Remedy and Natural Remedy from the Far East. Austria: Wilhelm Ennsthaler; 1995.

11. Junior RJ, Batista RA, Rodrigues SA, Filho LX, Lima AS. Antimicrobial activity of broth fermented with kombucha colonies. J Microb Biochem Technol 2009;1(1):72-8

12. Greenwalt CJ, Ledford RA, Streinkraus KH. Determination and characterization of the antimicrobial activity of the fermented tea kombucha. Leben Wissen Tech 1998;31:291-6.

13. Lu HJ, Breidt F Jr, Pérez-Díaz IM, Osborne JA. Antimicrobial effects of weak acids on the survival of Escherichia coli O157:H7 under anaerobic conditions. J Food Prot 2011;74:893-8.

14. Khatry S, Sirish KS, Shastri N, Sadanandam M. Novel drug delivery systems for antifungal therapy. Int J Pharm Pharm Sci 2010;2(4):6-9. 